\title{
Emotion and psychosis: links between depression, self-esteem, negative schematic beliefs and delusions and hallucinations
}

\author{
Ben Smith ${ }^{\mathrm{a}}$, David G. Fowler ${ }^{\mathrm{b}}$, Daniel Freeman ${ }^{\mathrm{c}}$, Paul Bebbington ${ }^{\mathrm{d}}$, \\ Hannah Bashforth ${ }^{\mathrm{c}}$, Philippa Garety ${ }^{\mathrm{c}}$, Graham Dunn ${ }^{\mathrm{e}}$ \& Elizabeth \\ Kuipers ${ }^{\mathrm{c}}$
}

${ }^{a}$ Royal Free \& University College London Medical School, UCL Department of Mental Health Sciences, Archway Campus, Holborn Union Building, Highgate Hill, London UK, N19 5LW; ben.smith@ucl.ac.uk Tel: 442072883024 (Author for correspondence)

${ }^{\mathrm{b}} \mathrm{School}$ of Medicine, Health Policy \& Practice, Elizabeth Fry Building, University of East Anglia, Norwich UK, NR4 7TJ; d.fowler@uea.ac.uk Tel: 441603593601 Fax: 441603593604

'King's College London, Department of Psychology, Box 77, Institute of Psychiatry, De Crespigny Park, London UK, SE5 8AF

${ }^{\mathrm{d}}$ Royal Free \& University College London Medical School, UCL Department of Mental Health Sciences, Bloomsbury Campus, Wolfson Building, 48, Riding House Street, London UK, W1W 7EY

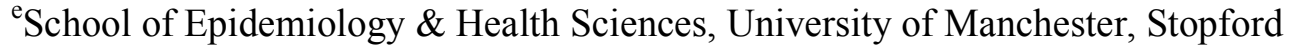
Building, Oxford Road Manchester UK, M13 9TT

\begin{abstract}
Background

Increasingly, the role of emotion in psychosis is being recognised. Cognitive conceptualisations of psychosis (e.g. Garety et al., 2001) emphasise a central, normal and direct (non-defensive) role for negative emotion in the development and maintenance of psychosis. This study tests specific predictions made by Garety et al (2001) about the role of emotion and negative evaluative beliefs in psychosis.
\end{abstract}




\section{Methods}

100 participants who had suffered a recent relapse in psychosis were recruited at baseline for the Prevention of Relapse in Psychosis (PRP) trial. In a cross-sectional analysis, we examined the role of depression, self-esteem and negative evaluative beliefs in relation to specific positive symptoms (persecutory delusions, auditory hallucinations and grandiose delusions) and symptom dimensions (e.g. distress, negative content, pre-occupation and conviction).

\section{Results}

Analysis indicated that individuals with more depression and lower self-esteem had auditory hallucinations of more intensely negative content and greater severity, and were more distressed by their auditory hallucinations. In addition, individuals with more depression, lower self-esteem and more negative evaluations about themselves and others had persecutory delusions of greater severity and were more pre-occupied and distressed by their delusions. Individuals with grandiose delusions of greater severity had less depression, higher self-esteem and less negative evaluations about themselves.

\section{Conclusions}

This study provides evidence for the role of emotion in schizophrenia spectrumdisorders. Mood, self-esteem and negative evaluative beliefs should be considered when conceptualising psychosis and designing interventions.

\section{Keywords}

Schematic beliefs; hallucinations; delusions; depression; schizophrenia 


\section{Introduction}

The role of emotion in the development and maintenance of psychosis is receiving renewed attention (e.g. Birchwood, 2003; Freeman \& Garety, 2003; Guillem et al., 2005; Hafner et al., 2005). There is now a body of evidence accumulating from epidemiological, questionnaire, experimental and treatment studies that low mood, low self-esteem and negative schematic beliefs can contribute to the development of psychotic symptoms (e.g. Barrowclough et al., 2003; Bowins \& Shugar 1998; Close \& Garety, 1998; Drake et al., 2004; Freeman et al., 1998; Freeman et al., 2003; Guillem et al., 2005; Hafner et al., 2005; Hall \& Tarrier, 2002; Iqbal et al., 2000; Krabbendam et al., 2002; Martin \& Penn 2001; Trower \& Chadwick 1995 and Van Os, 2000).

Despite these developments it remains unclear how schematic beliefs and emotional dysfunction interact in psychosis. Using a large sample the current study aims to test predictions made by Garety et al (2001) about the role of emotion and schematic beliefs in psychosis. This study builds on previous important work (e.g. Barrowclough et al, 2003) by extending analysis to individual symptoms and to symptom dimensions.

The empirical evidence so far is strong. Van Os (2000) reports a study of over 7000 individuals screened for psychiatric status and followed up for 3 years. Those who subsequently developed psychosis were found more likely to have low self-esteem and depressive schemas. 
Barrowclough et al. (2003) assessed negative self-evaluation using an in-depth interview in a group with schizophrenia $(\mathrm{N}=59)$. They found that negative selfevaluation was strongly associated with the positive symptoms of psychosis (PANSS positive sub-scale). Importantly, this remained significant when levels of depression were controlled.

Recent cognitive conceptualisations of psychosis (e.g. Bentall et al., 1994; Chadwick \& Birchwood, 1994; Garety et al., 2001 and Trower \& Chadwick, 1995) differ in their account of the role of emotion in psychosis. Bentall and colleagues understood persecutory delusions to be the result of a psychological defence against underlying negative emotion and low self-esteem (e.g. Bentall et al., 1994). In contrast, Garety and colleagues claim that negative emotion and low self-esteem have a central, normal and direct (non-defensive) role in the development of symptoms (Fowler, 2000; Freeman \& Garety, 2003; Garety, et al., 2001).

Garety et al (2001) propose a cognitive conceptualisation of psychosis. Building on the work of other researchers, (e.g. Maher, 1988; Frith, 1992; Hemsley, 1993; Bentall et al, 1994; Chadwick and Birchwood, 1994; Morrison et al, 1995) they propose that in the context of anomalous conscious experiences (e.g. heightened perceptions, thoughts experienced as voices) and adverse life events, emotional changes occur. Such emotional changes feed back into moment-by-moment processing of anomalous experiences, influence their content and perpetuate their occurrence. Fowler (2000) specifically suggests that distressing voices and persecutory delusions are associated with the appraisal of negative beliefs and thinking. For example, the content of 
distressing auditory hallucinations often mirror the content of depressive thinking associated with low mood (Fowler et al., 2000).

Garety et al (2001) also propose that these emotional processes occur against an important social cognitive background. Early adverse experiences are proposed to create an enduring cognitive vulnerability, characterised by negative schematic models of the self and others (e.g. I am vulnerable, others are dangerous). Fowler (2000) suggests that the triggering of negative schematic beliefs in individuals vulnerable to psychosis may lead to them hearing voices with threatening or critical content. Such a view suggests that it is also the accessing of negative schematic beliefs and thoughts of negative content, rather than simply depressed mood or low self-esteem that is associated with distressing voices and persecutory delusions.

In order to examine these proposals empirically we have developed a new measure of schematic beliefs in psychosis. The Brief Core Schema Scales (BCSS); (Fowler et al., In Press with Psychological Medicine) assess strongly held negative evaluations of self (e.g. I am weak, I am bad, I am useless) and strongly held negative evaluations of others (e.g. Others are untrustworthy, others are dishonest, others are threatening). The reason for developing this new measure is two-fold. First, there is a need for clinically relevant, quick self-report measures of schematic beliefs (of self and others) in large studies involving multiple assessments. The BCSS is clinically derived and aims to capture what is often reported by clients to their therapists. Secondly, we wanted to make clear distinctions between self-report measures of self-esteem (e.g. Rosenburg, 1965) and of schematic beliefs. The items in the BCSS measure strongly held negative self-evaluations and provide an operational construct of negative 
schematic self-beliefs which is distinct from existing assessments of self-esteem (that tend to measure presence of positive evaluations of self or their absence).

Hence, in this study (in accordance with Garety et al, 2001) we predicted normal, direct and non-defensive associations between individual symptoms (and symptom dimensions) and depression, low self-esteem and negative schematic beliefs. We first predicted that the severity, and distress/negative content dimensions, of auditory hallucinations would be associated with depression and low self-esteem. This is consistent with suggestions that the content of distressing auditory hallucinations specifically mirror the content of depressive thinking associated with low mood (Fowler et al, 2000). The direction of the relationship between auditory hallucinations and low mood/low self-esteem was predicted to be driven both by the auditory hallucinations and the resultant negative affect; a vicious cycle.

Our second prediction was that while there would be associations between low mood, low self-esteem and the severity, and distress dimensions, of persecutory delusions, negative evaluative beliefs about self and others would be independently associated with persecutory delusions once the confounding effects of depression and selfesteem were controlled. This would support theoretical proposals (e.g. Fowler, 2000; Garety et al, 2001 and Birchwood, 2003) and empirical evidence (e.g. Barrowclough et al, 2003) indicating a role for schematic beliefs in psychotic symptoms independent of mood.

Garety et al (2001) do not make specific predictions about the role of emotion in grandiose beliefs although Freeman \& Garety (2003) hypothesise that expansive or 
elated mood could build upon pre-existing inflated (or accurate) perceptions of self. This positive mood state might then reinforce and amplify aspects of the self-concept resulting in a grandiose belief. Smith et al. (2005) present data that support this view. Our third prediction was therefore that grandiose delusions would be associated with the absence of depressed mood and with elevated or positive views of self (selfesteem), a pattern of results we have found in our non-clinical study (Fowler et al., In Press). More speculatively we also hypothesised that grandiose delusions in a clinical sample would be combined with negative evaluative beliefs about others. Our reasoning for this was that a combination of elevated mood and positive views of self with negative evaluations of others may foster a social position that maintains positive self-evaluations, whilst ignoring social cues, thus leading to the maintenance of grandiose delusions.

\section{Methods}

The Psychological Prevention of Relapse in Psychosis (PRP) Trial is a British multicentre randomised controlled trial of cognitive behavioural therapy and family intervention for psychosis (ISRCTN83557988). The detailed methods of the PRP trial are published elsewhere (Freeman et al., 2004). For the present study, participants were assessed before randomisation to trial conditions took place.

\subsection{Participant criteria}

Participants were recruited within 3 months of a relapse in positive symptoms and had a current diagnosis of non-affective psychosis (schizophrenia, schizoaffective disorder, delusional disorder) (ICD-10, F20). Participants were aged 18-65 years and 
were rated at least 4 (moderate severity) on the PANSS on at least one positive psychotic symptom at initial interview.

The study was based upon data from the first 100 participants entering the PRP trial who completed the self-esteem measure at baseline $(77 \%$ of the total inducted at that time).

\subsection{Positive symptom measures}

Scale for the Assessment of Positive Symptoms (SAPS; Andreasen, 1984). The SAPS is a 35-item, 6 point (0-5) rating instrument. Symptoms are rated over the last month. The SAPS can be broken down into persecutory delusions, auditory hallucinations and grandiose delusions.

Positive and Negative Syndrome Scale (PANSS; Kay, 1991). The PANSS is a 30item, seven point (1-7) rating instrument of the phenomena associated with schizophrenia. Symptoms over the last 72 hours are rated. The PANSS was included to allow comparisons with Barrowclough, et al., 2003.

Psychotic Symptom Rating Scales (Haddock et al., 1999). The PSYRATS is a 17item, 5-point scale (0-4) multidimensional measure of delusions (6 items) and auditory hallucinations (11 items). All delusion items and six items from the auditory hallucinations scale were used. Excluded items overlapped with other positive symptom measures (e.g. frequency, location, duration, loudness and beliefs about origin of auditory hallucinations). Symptoms over the last week are rated. 


\subsection{Emotion and Belief measures}

Beck Depression Inventory - II (Beck et al., 1996). The BDI-II is a self-report 21item, four point scale (0-3) for the assessment of depression. Depression is assessed over the past fortnight.

Rosenberg Self-esteem Scale (Rosenberg, 1965). The RSES is a 10-item, four point scale (1-4) that assesses current levels of global self-esteem.

Brief Core Schema Scale (Fowler et al., In Press). The BCSS is a 24-item, five point rating scale (0-4) that assesses evaluative beliefs about the self and others. Four scores are obtained: negative self ( 6 items), positive self ( 6 items), negative others (6 items) and positive others (6 items). The negative self-scale is called the BCSS-NS and the negative other scale the BCSS-NO. The BCSS is a quick self-report measure.

The detailed psychometric properties of the BCSS are documented elsewhere (Fowler et al., In Press with Psychological Medicine). The BCSS showed good internal consistency and Cronbach's alpha coefficients were all $>0.78$. Principal components analysis revealed a four component solution consistent with the four sub-scale scores accounting for $57 \%$ of the variance.

\subsection{Analyses}

Analyses were conducted using SPSS for windows (version 11.5), (SPSS, 2003) and Stata (release 8.0), (StataCorp, 2003). 


\section{Results}

\subsection{Demographic and clinical data}

Sixty eight percent of the sample was male and the mean age was 39 years $(\mathrm{SD}=10.9)$, range 19-65. Sixty nine percent had been admitted to hospital as a result of their recent relapse in psychosis. Almost 70\% described themselves as White-British, 10\% as Black-Caribbean, $7 \%$ as Black-African and $11 \%$ as from other ethic backgrounds. Seventy eight percent had a diagnosis of schizophrenia, $20 \%$ of schizoaffective disorder and $2 \%$ of delusional disorder. The mean length of illness was 11.7 years $(\mathrm{SD}=10.1)$, with a range from less than 1 year up to 44 years.

The mean scores for the psychotic symptom measures are presented in Table 1. Fifty five percent of the sample reported persecutory delusions, 57\% auditory hallucinations, and 17\% grandiose delusions (all rated moderate-severe on the SAPS). Self-esteem, depression and negative evaluative belief scores are also presented in Table 1.

Table 1. about here

\subsection{The relationship between depression, self-esteem and negative evaluative beliefs}

BDI, RSES and BCSS-NS scores were strongly associated with one-another (see Table 2). Associations between BCSS-NO and BDI, RSES and BCSS-NS were moderate.

Table 2. about here 
3.3 The relationship between global positive symptoms, depression, self-esteem and negative evaluative beliefs

The two global measures of positive symptoms (SAPS and PANSS positive subscale) were strongly associated $(\mathrm{r}=.71, \mathrm{p}<.001)$.

There were no significant associations between SAPS total score and the BDI $(\mathrm{r}=.12$, $\mathrm{p}=.14)$, RSES $(\mathrm{r}=.10, \mathrm{p}=.19)$ and BCSS-NS $(\mathrm{r}=.10, \mathrm{p}=.18)$. An association was identified, however, between SAPS total score and BCSS-NO $(r=.34, \mathrm{p}=.001)$.

There were similarly no significant associations with the PANSS positive sub-scale; BDI $(\mathrm{r}=.09, \mathrm{p}=.34)$, RSES $(\mathrm{r}=.08, \mathrm{p}=.41)$ and BCSS-NS $(\mathrm{r}=.14, \mathrm{p}=.18)$. Again, however, an association was identified between PANSS positive sub-scale and BCSS$\mathrm{NO}(\mathrm{r}=.38, \mathrm{p}<.001)$.

3.4 The relationship between specific positive symptoms, depression, self-esteem and negative evaluative beliefs

For the analysis of specific positive symptoms the individual items from the SAPS were used. Persecutory delusions were strongly associated with depression, low selfesteem and negative evaluative beliefs about self, although the association with negative evaluative beliefs about others was weaker than expected. Auditory hallucinations were also strongly associated with both depression and low self-esteem but were not associated with negative evaluative beliefs about self or others. Grandiose delusions were inversely associated with depression, low self-esteem and negative evaluative beliefs about self but had no association with negative evaluative beliefs about others (See Table 3). 


\section{Table 3. about here}

Multivariate analyses were utilised to tease apart the relative contributions of depression, self-esteem and negative evaluative beliefs to individual symptoms.

Ordered logistic regression revealed that negative evaluative beliefs about self was the only variable to be independently and significantly associated with persecutory delusions (See Table 4).

Ordered logistic regression revealed that depression was the only variable to be independently associated with auditory hallucinations. This result failed to reach significance at the $95 \%$ interval but was an appreciable trend $(p=.067)$, (See Table 4$)$.

Ordered logistic regression also revealed that both depression (inversely) and negative evaluative beliefs about others were independently and significantly associated with grandiose delusions (See Table 4).

\section{Table 4. about here}

\subsection{Positive symptom dimensions, depression, self-esteem and negative evaluative} beliefs

Individuals who were more depressed, had lower self-esteem and more negative evaluative beliefs about themselves and others were also more pre-occupied and distressed by their delusions. The more depressed individuals were also more convinced of their delusions (although this relationship was weaker) and reported more disruption to their lives (see Table 5). 


\section{Table 5. about here}

Individuals who were more depressed, had lower self-esteem and more negative evaluative beliefs about themselves were more distressed by their auditory hallucinations. They also had more auditory hallucinations of negative content and with a higher degree (intensity) of negative content. Those who were more depressed and had lower self-esteem also reported more disruption to their lives and that their auditory hallucinations were less controllable. Negative evaluative beliefs about others were not associated with any of the measured dimensions of auditory hallucinations. (see Table 6).

\section{Table 6. about here}

\section{Discussion}

The current findings are consistent with the existing empirical evidence (e.g. Van Os, 2000) that low mood, low self-esteem and negative schematic beliefs can contribute to the development of psychotic symptoms. Our prediction of normal, direct and nondefensive associations between individual symptoms (and symptom dimensions) and depression, low self-esteem and negative schematic beliefs has been supported.

We first predicted that the severity, and distress/negative content dimensions, of auditory hallucinations would be associated with depression and low self-esteem. Individuals with more depression and lower self-esteem (but not more negative evaluative beliefs about themselves or others) were found to have auditory 
hallucinations of greater severity. Furthermore, individuals who were more depressed, had lower self-esteem and more negative evaluative beliefs about themselves were more distressed by their auditory hallucinations. They also had more auditory hallucinations of negative content and with a higher degree (intensity) of negative content. There was also a trend for depression to be independently associated with auditory hallucinations. We speculate like others (e.g. Chadwick \& Birchwood, 1994) that the direction of the relationship between auditory hallucinations and low mood is driven both by the auditory hallucinations and the resultant negative affect; a vicious cycle. In other words, distressing auditory hallucinations with a negative content have an immediate and powerful impact on mood, and low mood in turn can make an individual vulnerable to further auditory hallucinations.

Our second prediction was that negative evaluative beliefs about self and others would be independently associated with persecutory delusions once the confounding effects of depression and low self-esteem were controlled. Importantly, we found that negative evaluative beliefs about self (but not others) were associated with persecutory delusions independent of current mood and self-esteem. This supports both theoretical proposals (e.g. Fowler, 2000; Garety et al, 2001 and Birchwood, 2003) and empirical evidence (e.g. Barrowclough et al, 2003) indicating a role for schematic beliefs in psychotic symptoms independent of mood.

Our third prediction was that grandiose delusions would be associated with the absence of depressed mood and with elevated or positive views of self (self-esteem). We found that individuals with grandiose delusions of greater severity had less depression, higher self-esteem and less negative evaluations about themselves. More 
speculatively we had also hypothesised that grandiose delusions would be combined with negative evaluative beliefs about others. Bi-variate analysis revealed no such associations, although logistic regression indicated that depression (inversely) and negative evaluative beliefs about others were both independently associated with grandiose delusions. This suggests that grandiosity is linked to the accessing of positive self-beliefs (and possibly negative other-beliefs) and the resultant, and reinforcing, absence or lack of negative mood state (Freeman \& Garety 2003; Smith et al., 2005).

Hence, both the current study and that of Barrowclough et al. (2003) have found negative evaluative beliefs about self to be independently and significantly associated with psychotic symptoms. Barrowclough et al. (2003) found an independent association with global positive symptoms. In our study we found an independent association with persecutory delusions but not with auditory hallucinations, grandiose delusions or global positive symptoms. Our result should be interpreted tentatively. One explanation is that there is an independent and direct role for negative evaluative self-beliefs in the development of psychotic symptoms. As Garety et al (2001) have proposed, underlying negative schemas such as "I am weak" might influence information processing and lead to a current sense of threat in the context of anomalous psychotic experience or adverse life events, resulting in delusional explanations of these experiences.

However because of the cross-sectional nature of our design, there is an alternative explanation. The negative evaluative beliefs may not be causal in psychosis, rather they may be a consequence of it. When measuring schemas in psychosis the 
information we gather is influenced by the current and recent experience of psychosis itself, by current levels of threat and by current emotional dysfunction. We can only conclude that negative evaluative beliefs about self are associated with psychosis. It simply remains unclear how they are associated.

\subsection{Limitations}

Participants had all consented to a treatment trial and may therefore not be fully representative of those with psychosis. Measures were taken following a relapse of psychosis and may therefore not be stable over time. Our new measure (BCSS) requires further use and psychometric analysis. Only overt self-esteem has been measured. Future studies should consider measuring of covert self-esteem across time

(see Bentall ????). As with any cross-sectional design, the evolution and direction of associations cannot be ascertained.

\subsection{Implications}

In order to understand how negative evaluative beliefs relate to psychosis (i.e. underlying beliefs or co-occurring beliefs) it is essential to measure them across time. Do they remain stable or change in response to changes in psychosis? A follow up study is planned to address this issue in the full PRP sample when the data becomes available. This will also allow us to examine in more detail (and with more power) the role other variables may play in influencing the relationships between emotion and psychosis (e.g. length of illness).

This study provides broad support for psychological interventions in psychosis that address emotional dysfunction (e.g. cognitive behavioural therapy and family 
intervention). Fowler (2000) notes that individuals with psychosis are often not aware of the meaningful links between their symptoms, life experiences, mood and beliefs. Helping someone understand his or her problem as partly one of belief and interpretation, rather than actual and current threat, can be beneficial. Clinicians can adapt their formulation and treatment skills from cognitive behavioural therapy for non-psychotic disorders to their work in psychosis. There is evidence here that one clue to the development and maintenance of psychotic symptoms is that normal and understandable negative emotional processes are at work.

\section{Acknowledgements}

We would like to thank all the participants who took part in the study from both London and East Anglia and all the staff who helped in recruitment.

\section{Declaration of Interest}

This study was supported by a Wellcome Trust Programme Grant: 062452. PRP is registered as ISRCTN: 83557988 .

\section{References}

Andreasen, N.C. (1984). The Scale for the Assessment of Positive Symptoms (SAPS). Iowa City, IA: The University of Iowa.

Barrowclough, C., Tarrier, N., Humphreys, L., Ward, J., Gregg, L. \& Andrews, B. (2003). Self-esteem in schizophrenia: relationships between self-evaluation, family attitudes and symptomology. Journal of Abnormal Psychology, 112(1), 92-99. 
Beck, A.T., Steer, R.A., \& Brown, G.K. (1996). BDI-II Manual. The Psychological Corporation: San Antonia.

Bentall, R.P., Kinderman, P. \& Kaney, S. (1994). The self, attribution processes and abnormal beliefs: Towards a model of persecutory delusions. Behaviour Research and Therapy, 32, 331-341.

Birchwood, M. (2003). Pathways to emotional dysfunction in first-episode psychosis. British Journal of Psychiatry, 182, 373-375.

Bowins, B. \& Shugar, G. (1998). Delusions and self-esteem. Canadian Journal of Psychiatry, 43, 154-158.

Chadwick, P. D. J. \& Birchwood, M, J. (1994). The omnipotence of voices: A cognitive approach to hallucinations. British Journal of Psychiatry, 164, 190-201.

Close, H. \& Garety, P.A. (1998). Cognitive assessment of voices: further developments in understanding the emotional impact of voices. British Journal of Clinical Psychology, 37, 173-188.

Drake, R.J, Pickles, A., Bentall, R.P., Kinderman, P., Haddock, G., Tarrier, N. \& Lewis, S.W. (2004). The evolution of insight, paranoia and depression during early schizophrenia. Psychological Medicine, 34, 285-292. 
Fowler, D. (2000). Cognitive behaviour therapy for psychosis: from understanding to treatment. Psychiatric Rehabilitation Skills, 4(2), 199-215.

Fowler, D.G., Freeman, D., Smith, B., Garety, P.A., Kuipers, E.K., Bebbington, P.E. \& Dunn, G. The brief core schema scales: psychometric properties and associations with paranoia, depression, and anxiety in normal and psychosis samples. In press with Psychological Medicine.

Freeman, D. \& Garety, P.A. (2003). Connecting neurosis and psychosis: the direct influence of emotion on delusions and hallucinations. Behaviour Research and Therapy, 41 (8), 923-947.

Freeman, D., Garety, P.A., Fowler, D., Kuipers, E.K., Dunn, G., Bebbington, P. \& Hadley, C. (1998). The London-East Anglia randomised controlled trial of cognitivebehaviour therapy for psychosis IV: Self-esteem and persecutory delusions. British Journal of Clinical Psychology, 37, 415-430.

Freeman, D., Garety, P.A., Fowler, D., Kuipers, E., Bebbington, P.E., Dunn, G. (2004). Why Do People With Delusions Fail to Choose More Realistic Explanations for Their Experiences? An Empirical Investigation. Journal of Consulting \& Clinical Psychology, 72, $671-680$.

Freeman, D., Slater, M., Bebbington, P.E., Garety, P.A., Kuipers, E.K., Fowler, D., Met, A., Read, C., Jordan, J. \& Vinayagamoorthy, V. (2003). Can virtual reality be 
used to investigate persecutory ideation? The Journal of Nervous and Mental Disease, 191, 509-514.

Garety, P.A., Kuipers, E.K., Fowler, D., Freeman, D. \& Bebbington, P.E. (2001). A cognitive model of the positive symptoms of psychosis. Psychological Medicine, 31, 189-195.

Garety, P.A., Freeman, D., Jolley, S., Dunn, G., Bebbington, P.E., Fowler, D., Kuipers, E. \& Dudley, R. (2005). Reasoning, Emotions and Delusional Conviction in Psychosis. Journal of Abnormal Psychology, 114, 373-384.

Guillem, F., Pampoulova, T., Stip, E., Lalonde, P. \& Todorov, C. (2005). The relationship between symptom dimensions and dysphoria in schizophrenia. Schizophrenia Research, 75, 83-96.

Haddock, G., McCarron, J., Tarrier, N., \& Faragher, E.B. (1999). Scales to measure dimensions of hallucinations and delusions: the psychotic symptom rating scales (PSYRATS). Psychological Medicine, 29, 879-889.

Hafner, H., Maurer, K., Trendler, G., an der Heiden, W., Schmidt, M. \& Konnecke, R. (2005). Schizophrenia and depression: Challenging the paradigm of two separate diseases - A controlled study of schizophrenia, depression and healthy controls. Schizophrenia Research, 77, 11-24. 
Hall, P.l. \& Tarrier, N. (2003). The cognitive behavioural treatment of low selfesteem in psychotic patients: a pilot study. Behaviour Research and Therapy, 41(3), $317-322$.

Iqbal, Z., Birchwood, M., Chadwick, P. \& Trower, P. (2000). Cognitive approach to depression and suicidal thinking in psychosis: 2. Testing the validity of a social ranking model. British Journal of Psychiatry, 177, 522-528.

Kay, S. R. (1991). Positive and negative syndromes in schizophrenia: Assessment and research. Brunner/Mazel: New York.

Krabbendam, L., Janssen, I., Bijl, R.V., Vollerburgh, W.A.M. \& van Os, J. (2002). Neoroticism and low self-esteem as risk factors for psychosis. Social Psychiatry and Psychiatric Epidemiology, 37, 1-6.

Martin, J.A. \& Penn, D.L. (2001). Brief Report: social cognition and subclinical paranoid ideation. British Journal of Clinical Psychology, 40, 261-265.

Rosenberg, M. (1965). Society and the Adolescent Self-Image. Princeton University Press: Princeton.

Smith, N., Freeman, D., \& Kuipers, E., (2005). Grandiose delusions: an experimental investigation of the delusion-as-defence hypothesis. Journal of Nervous and Mental Disease, 193, 480-487. 
StataCorp (2003). Stata statistical software: release 8.0. Stata Corporation: College Station, TX.

Trower, P. \& Chadwick, P. D. J. (1995). Pathways to defence of the self: A theory of two types of paranoia. Clinical Psychology: Science and Practice, 2, 263-278.

Zimmermann, G., Favrod, J., Triieu, V.H. \& Pomini, V. (2005). The effect of cognitive behavioural treatment on the positive symptoms of schizophrenia spectrum disorders: A meta-analysis. Schizophrenia Research, 77, 1-9. 


\begin{tabular}{lll}
\hline & Mean & S.D. \\
PANS positive sub-scale & 18.52 & 5.23 \\
SAPS total score & 7.32 & 3.07 \\
SAPS persecutory delusions & 2.19 & 1.78 \\
SAPS grandiose delusions & 0.98 & 1.47 \\
SAPS auditory hallucinations & 2.55 & 2.01 \\
PSYRATS auditory hallucinations & 18.34 & 14.49 \\
PSYRATS delusions & 14.11 & 6.59 \\
RSES total score & 23.79 & 6.76 \\
BDI total score & 23.98 & 13.44 \\
BCSS-NS & 7.92 & 5.93 \\
BCSS-NO & 10.16 & 6.81 \\
\hline
\end{tabular}

Table 1: Means and standard deviations for all variables $(\mathrm{N}=100)$ 


\begin{tabular}{llll}
\hline & RSES & BCSS-NS & BCSS-NO \\
\hline BDI & $.73, \mathrm{p}<.001$ & $.62, \mathrm{p}<.001$ & $.42, \mathrm{p}<.001$ \\
RSES & & $.70, \mathrm{p}<.001$ & $.29, \mathrm{p}=.006$ \\
BCS-NS & & $.52, \mathrm{p}<.001$ \\
\hline
\end{tabular}

Table 2: Pearson's bi-variate correlations (two-tailed probabilities) between depression, self-esteem and negative evaluative beliefs 


\begin{tabular}{lllll}
\hline Symptom & BDI & RSES & BCSS-NS & BCSS-NO \\
\hline $\begin{array}{l}\text { Persecutory } \\
\text { Delusions }\end{array}$ & $.43, p<.001$ & $.36, p<.001$ & $.41, p<.001$ & $.22, p=.041$ \\
$\begin{array}{l}\text { Auditory } \\
\text { Hallucinations }\end{array}$ & $.32, p=.001$ & $.32, p=.001$ & $.17, p=.099$ & $.03, p=.785$ \\
$\begin{array}{l}\text { Grandiose } \\
\text { Delusions }\end{array}$ & $-.38, p<.001$ & $-.33, p=.001$ & $-.30, p=.004$ & $.09, p=.393$ \\
\hline
\end{tabular}

Table 3: Spearman's bi-variate correlations (two-tailed probabilities) between specific positive symptoms (SAPS items), depression, self-esteem and negative evaluative beliefs 


\begin{tabular}{|c|c|c|c|c|}
\hline Symptom/Independent Variable & Coefficient (odds ratio) & Std. Err. & $\mathrm{Z}$ & $\mathrm{P}$ \\
\hline \multicolumn{5}{|l|}{ Persecutory Delusions } \\
\hline Self-esteem & -.007 & .054 & -.14 & .886 \\
\hline BCSS-NO & -.019 & .037 & -.51 & .607 \\
\hline \multicolumn{5}{|l|}{ Auditory Hallucinations } \\
\hline BCSS-NS & .024 & .054 & .44 & .660 \\
\hline BCSS-NO & -.035 & .036 & -.99 & .322 \\
\hline \multicolumn{5}{|l|}{ Grandiose Delusions } \\
\hline Depression & -.108 & .037 & -2.89 & .004 \\
\hline Self-esteem & -.015 & .060 & -.25 & .804 \\
\hline
\end{tabular}

Table 4: Ordered ologit estimations (logistic regression) for specific positive symptoms 


\begin{tabular}{lllll}
\hline Delusion Dimension & BDI & RSES & BCSS-NS & BCS-NO \\
\hline Amount of pre-occupation & $.25, p=.016$ & $.14, p=.157$ & $.23, p=.032$ & $.31, p=.004$ \\
Duration of pre-occupation & $.47, p<.001$ & $.30, p=.003$ & $.25, p=.016$ & $.33, p=.002$ \\
Conviction & $.21, p=.046$ & $.10, p=.312$ & $.09, p=.378$ & $.23, p=.039$ \\
Amount of distress & $.47, p<.001$ & $.40, p<.001$ & $.38, p<.001$ & $.22, p=.041$ \\
Intensity of distress & $.39, p<.001$ & $.29, p=.004$ & $.24, p=.021$ & $.17, p=.121$ \\
Disruption to life & $.24, p=.019$ & $.10, p=.306$ & $.11, p=.322$ & $.16, p=.144$ \\
\hline
\end{tabular}

Table 5: Spearman's bi-variate correlations (two-tailed probabilities) between dimensions of delusions (PSYRATS), depression, self-esteem and negative evaluative beliefs 


\begin{tabular}{lllll}
\hline $\begin{array}{l}\text { Hallucination } \\
\text { Dimension }\end{array}$ & BDI & RSES & BCSS-NS & BCSS-NO \\
\hline $\begin{array}{l}\text { Amount of negative } \\
\text { content }\end{array}$ & $.50, p<.001$ & $.38, p<.001$ & $.31, p=.003$ & $.15, p=.176$ \\
$\begin{array}{l}\text { Degree of negative } \\
\text { content }\end{array}$ & $.43, p<.001$ & $.38, p<.001$ & $.36, p=.001$ & $.16, p=.151$ \\
Amount of distress & $.42, p<.001$ & $.29, p=.005$ & $.22, p=.035$ & $.08, p=.459$ \\
$\begin{array}{l}\text { Intensity of distress } \\
\text { Disruption to life }\end{array}$ & $.43, p<.001$ & $.30, p=.003$ & $.21, p=.044$ & $-.03, p=.787$ \\
Controllability & $.34, p=.001$ & $.29, p=.004$ & $.13, p=.226$ & $-.02, p=.831$ \\
\end{tabular}

Table 6: Spearman's bi-variate correlations (two-tailed probabilities) between dimensions of auditory hallucinations (PSYRATS), depression, self-esteem and negative evaluative beliefs 